\title{
Integrin a5 $\beta 1$-Ang1/Tie2 receptor cross-talk regulates brain endothelial cell responses following cerebral ischemia
}

\author{
Defang Pang ${ }^{1}$, Lu Wang ${ }^{2,3}$, Jing Dong ${ }^{4}$, Xiaoyin Lai ${ }^{2}$, Qijuan Huang ${ }^{5}$, Richard Milner ${ }^{6}$ and Longxuan Li ${ }^{2}$
}

\begin{abstract}
We have previously demonstrated that in response to cerebral ischemia (Cl), the growth factor angiopoietin-1 (Ang1) and a5 $\beta 1$ integrin are both induced in cerebral vessels, which likely provide positive signals driving the endogenous angiogenic response and vascular protection after $\mathrm{Cl}$. However, the precise relationship between endothelial Ang1 and $a 5 \beta 1$ integrin after $\mathrm{Cl}$ remains poorly understood. Here, we investigated the effects of the interaction between the Ang1/Tie2 system and a5 $\beta 1$ integrin on brain endothelial cells (BECS) under cerebral ischemic conditions in vivo and in vitro. Immunofluorescence analysis demonstrated that integrin a5 31 co-localized with Tie2/phosphorylated Tie2 on cerebral vessels in the penumbra. The in vitro study showed that oxygen-glucose deprivation/restoration (OGD/R) induced the expression of the Ang1 receptor Tie2 on BECs in a manner similar to that for integrin a5 and Ang1 in response to OGD/R, accompanied by increased activation of Tie2 and its downstream effectors focal adhesion kinase (FAK) and Akt. Knockdown of a5 integrin markedly suppressed OGD/R-induced Tie2 receptor activation in BECs, while in contrast, priming BECs with Ang1 promoted the expression of a5 integrin as well as the Tie2 downstream transcription factor Ets-1 in OGD-treated BECs. In line with this, Ets-1 knockdown significantly attenuated Ang1mediated upregulation of a5 integrin. Functionally, Ang1 induced cell migration and tube formation of BECs after OGD, but this effect was inhibited by diminishment of the levels of a5 integrin in BECs. Taken together, our data indicate that the Ang1/Tie2 system cross-talks with integrin a5 $\beta 1$ in BECs after $\mathrm{Cl}$, which may contribute to the endogenous angiogenic vascular protective response following $\mathrm{Cl}$.
\end{abstract}

\section{Introduction}

Angiogenic remodeling has been described in the penumbral regions in animal models of stroke as well as in the ischemic brains of stroke patients ${ }^{1-4}$. Greater microvessel density in the ischemic border has been reported to correlate with longer survival in stroke patients ${ }^{4}$. It is commonly believed that angiogenic

Correspondence: Richard Milner (rmilner@scripps.edu) or

Longxuan Li (Longxuanlee2006@yahoo.com)

${ }^{1}$ Department of Special Outpatient Service, Gongli Hospital, The Second

Military Medical University, Shanghai 200135, P. R. China

${ }^{2}$ Department of Neurology, Gongli Hospital, The Second Military Medical

University, Shanghai 200135, P. R. China

Full list of author information is available at the end of the article.

These authors contributed equally: Defang Pang, Lu Wang remodeling may enhance cerebral perfusion, which leads to improved functional recovery after stroke ${ }^{5}$.

Angiopoietin-1 (Ang1) is an endogenous ligand for the vascular endothelial receptor tyrosine kinase Tie2. Mounting evidence shows that activation of Tie2 signaling pathways by Ang1 promotes endothelial cell/endothelial progenitor cell migration and sprouting, and protects the endothelial cells from apoptosis by phosphorylating the downstream effectors focal adhesion kinase (FAK) and Akt, a serine/threonine-specific protein kinase $^{6-8}$. Therefore, the Ang1/Tie2 system is considered as a promising molecular target for promoting therapeutic neovascularization and vascular protection.

In a recent study, we observed that in response to cerebral ischemia, Ang1 and the pro-angiogenic factor $\alpha 5 \beta 1$ 
integrin were both induced in cerebral vessels and brain endothelial cells (BECs) in vivo and in vitro ${ }^{1,9}$. Moreover, extensive co-localization of Ang1 with $\alpha 5$ integrin was found on angiogenic blood vessels in the ischemic penumbra ${ }^{9}$. Interestingly, BEC proliferation and tight junction protein expression followed the same time course as the expression of $\alpha 5 \beta 1$ and Ang1, suggesting a close correlation between a5 integrin and Ang1 in promoting angiogenesis and tight junction formation in the blood-brain barrier (BBB) after ischemic stroke.

Tie2/ $\alpha 5 \beta 1$ interactions modulate Ang-1-triggered signaling pathways in endothelial cells ${ }^{10}$. There is also evidence that stimulation of peripheral blood stem cells enriched by granulocyte colony-stimulating factor mobilization and apheresis (mobPBSCs) with cartilage oligomeric matrix protein (COMP)-Ang1 (a soluble, stable and potent Ang1 variant) induces the expression of $\alpha 5 \beta 1$ integrin $^{11}$. However, the precise relationship between the Ang1/Tie2 system and $\alpha 5 \beta 1$ integrin after cerebral ischemic stroke is poorly understood. The aim of this study was to investigate the cross-talk between the proangiogenic factor $\alpha 5 \beta 1$ integrin and Ang1 in BECs and the functional implications of this interaction in mediating $\mathrm{BEC}$ migration and tube formation under ischemic conditions in vivo and in vitro.

\section{Materials and methods \\ Experimental animals}

Male C57Bl/6 mice weighing $20-25 \mathrm{~g}$ at the time of surgery were used for all experiments. The present study was conducted in accordance with the NIH guidelines for the care and use of animals in research and under protocols approved by the Animal Care and Use Committee of Gongli Hospital, Pudong New Area, Shanghai.

\section{MCAO model}

Focal cerebral ischemia/ischemic stroke was induced by reversible right middle cerebral artery occlusion (MCAO) surgery under pentobarbital anesthesia, followed by reperfusion as described previously ${ }^{9}$. After $90 \mathrm{~min}$ of MCAO, the mice were briefly re-anesthetized and reperfusion was commenced. Sham animals were subjected to an identical procedure, but they did not undergo MCAO. Intra-ischemic neurological deficit was confirmed and scored as previously described ${ }^{2}$.

\section{Cell culture}

Immortalized mouse BECs of the bEnd3 cell line were obtained from the Shanghai Bioleaf Biotech Co., Ltd. Primary human brain microvascular endothelial cells (HBMECs) were purchased from Cell Systems (Kirkland, WA, USA). Cells (bEnd3 and HBMECs) were grown in six-well plates pre-coated with type I or IV collagen $\left(10 \mu \mathrm{g} / \mathrm{mL}\right.$, Sigma, for $2 \mathrm{~h}$ at $\left.37^{\circ} \mathrm{C}\right)$. The culture medium was endothelial basal medium (EBM-2) (Lonza, CC-3156) supplemented with 10\% FBS (Gibco), ascorbic acid, Lglutamine, penicillin/streptomycin, and human basic fibroblast growth factor (bFGF) (all from Sigma). Cells were maintained in a humidified incubator at $37^{\circ} \mathrm{C}$ and $5 \% \mathrm{CO} 2$, and the medium was changed every $48 \mathrm{~h}$.

\section{Oxygen-glucose deprivation and restoration (OGD/R)}

$\mathrm{BEC}$ cultures were subjected to ischemia-like injury through oxygen-glucose deprivation (OGD) for $4 \mathrm{~h}$ by placing the cultures in a deoxygenated glucose-free balanced salt solution (BSSO) in an anaerobic chamber (Forma, Thermo Scientific, Asheville, NC, USA) with an atmosphere of $5 \% \mathrm{CO}_{2}$ and $95 \% \mathrm{~N}_{2}$. After $4 \mathrm{~h}$ of OGD, the cultures were returned to control conditions (restoration) by adding $5.5 \mathrm{mM}$ glucose to the media under normoxic conditions. Control cultures (no injury) were incubated with a balanced salt solution containing $5.5 \mathrm{mM}$ glucose (BSS5.5). All cultures were maintained in a humidified $37^{\circ} \mathrm{C}$ incubator.

\section{Immunofluorescent studies and antibodies}

Mice at different time points of reperfusion were euthanized by perfusion with ice-cold saline, and then, the brains were rapidly dissected and stored at $-80^{\circ} \mathrm{C}$. Immunofluorescence (IF) studies were performed as previously described ${ }^{9}$ on 10 -mm-thick frozen coronal sections. The PE-conjugated rat anti-mouse $\alpha 5$ (CD49e) (clone 5H10-27, 1:150) antibody was purchased from BD Pharmingen (La Jolla, CA, USA), phospho-Tie2 (Tyr1108) polyclonal antibody (PA5-38339, 1:100) was obtained from Thermo Fisher Scientific (Rockford, IL, USA), and mouse anti-Tie2 antibody (AB24859, 1:25) was obtained from Abcam (Cambridge, MA, USA). The Cy3conjugated goat anti-rat secondary antibody was obtained from EarthOx. AlexaFluor 488-conjugated goat anti-mouse and rabbit secondary antibodies were purchased from Jackson Immunoresearch (West Grove, PA, USA). The negative controls for staining and confocal imaging were used to confirm the coexistence of vessel proteins.

\section{siRNA transfection}

GIPZ Lentiviral mouse Itga5 (Cat\# VGH5526EG16402), Ets-1 (Cat\# VGH5526-EG23871), small hairpin RNA (shRNA), and non-targeting shRNA controls were purchased from Dharmacon (Lafayette, CO, USA). $\mathrm{BECs}$, at a concentration of $2.4 \times 10^{5}$, were plated in sixwell plates in $2 \mathrm{~mL}$ of growth medium for $24 \mathrm{~h}$ prior to transfection. The following day, transfection was performed when the cells reached $70-80 \%$ confluence. shRNAs, including GIPZ Itga5, Ets-1, or non-silencing shRNA viral particle, were incubated with DharmaFECT $\mathrm{kb}$ transfection reagent (Lafayette, CO, USA), according 
to the manufacturer's reverse transfection protocol. After the medium was replaced with a new growth medium, transfection complexes were added to each well (final concentration of $2 \mu \mathrm{g} / \mathrm{mL}$ per well) and incubated at $37{ }^{\circ} \mathrm{C}$ in a $\mathrm{CO} 2$ incubator. Forty-eight hours after transfection, cells were subjected to OGD/R treatment with or without drug administration. Silencing of specific genes was confirmed by western blot.

\section{Drug administration}

After $4 \mathrm{~h}$ OGD, BECs were transferred to normal conditions to terminate the OGD and begin restoration. To detect the Ang1 effects on BEC $\alpha 5$ integrin expression/ $\alpha 5 \beta 1$ effects on BEC Tie2 signaling after OGD, control or shRNA Ets-1/shRNA $\alpha 5$-treated BECs were stimulated with or without Ang1 (50, 100, or $200 \mathrm{ng} / \mathrm{mL}$ ), as indicated for $15 \mathrm{~min}, 1 \mathrm{~h}$ or $12 \mathrm{~h}$ at the beginning of restoration. Cells were then harvested for western blotting at different time points after restoration initiation.

\section{Western blot analysis}

BECs were harvested and lysed with lysis buffer (1\% NP40, $50 \mathrm{mM}$ TrisHCl, $\mathrm{pH} 8.0,150 \mathrm{mM}$ sodium chloride) supplemented with protease and phosphatase inhibitor cocktails. Protein concentration was determined using the BCA protein assay kit (Eppendorf-Bio photometer, Germany). Western blotting and semi-quantitative analysis were performed as described previously ${ }^{9}$. The primary antibodies used were rabbit anti-angiopoietin-1 (AB10516, 1:1000, Merck Millipore, Darmstadt, Germany), rabbit anti- $\alpha 5$ (AB1928, 1:1000, Merck Millipore, Darmstadt, Germany), rabbit anti- $\alpha 5$ (\#4705, 1:1000, Cell Signaling Technology, Inc., Danvers, MA, USA), mouse anti-Ets-1 (sc-55581, 1:200, Santa Cruz Biotechnology, INC., USA), rabbit anti-phospho-Tie-2 (Y992) (AF2720, 1:400, R\&D Systems, Inc. Minneapolis, MN, USA), mouse anti-Tie2 (ab24859, 1:100, Abcam, Cambridge, MA, USA), rabbit anti-phospho-FAK (Tyr397) (ABT135,1:1000, EMD Millipore Corporation, Temecula, CA, USA), rabbit anti- FAK (06-543, EMD Millipore Corporation, Billerica, Massachusetts, USA 1:200), rabbit anti-phospho-Akt (Ser473) (9271 L, Cell Signaling Technology, Danvers, Massachusetts, USA 1:1000), rabbit antiAkt (9272 s, 1:1000, Cell Signaling Technology, Danvers, Massachusetts, USA), and $\beta$-actin (1:1000, Neomarker, Fremont, CA). For each sample, the protein levels were first normalized to the level of $\beta$-actin and then expressed as the fold increase over the level of the NO-OGD/R control group.

\section{Scratch wound migration assay}

BECs were transfected with the indicated shRNA, then seeded in six-well tissue culture plates $\left(5 \times 10^{5}\right.$ cells per well), and cultured to confluence. The confluent monolayer cells were subjected to OGD for $4 \mathrm{~h}$ and then scratched using a sterile $200-\mu \mathrm{L}$ pipette tip. The remaining wounded monolayer was washed twice with PBS to clear the cell debris. The BECs were re-fed with mitomycin C (1 mM, Sigma-Aldrich) containing serum-free EBM-2 or serum-free EBM-2 containing Ang1 (100 ng/ $\mathrm{mL}$ ) and then left to heal for $24 \mathrm{~h}$ at normoxia. Photographs were taken at the same site at 0 and $24 \mathrm{~h}$ after the injury. The healing of the wounds was assessed by measuring the wound gap. The experiments were undertaken in triplicate and were blindly analyzed.

\section{Tube formation assay}

BECs were transfected with the indicated shRNA and then exposed to OGD for $4 \mathrm{~h}$. The in vitro tube formation assay was carried out as previously described ${ }^{12,13}$. In brief, $250 \mu \mathrm{L}$ of growth factor-reduced Matrigel (BD Biosciences, San Diego, CA) was transferred to a well of a 24well tissue culture plate and polymerized for $30 \mathrm{~min}$ at $37^{\circ} \mathrm{C}$. BECs $\left(1 \times 10^{5}\right.$ cells/well $)$ were plated onto the layer of Matrigel in the presence or absence of Ang1 (100 ng/ $\mathrm{mL}$ ) as indicated. Twelve hours later, four representative fields were examined, and the average total areas of complete tubes formed by cells per unit area were compared. This examination was repeated three times and was blindly analyzed.

\section{Statistical analysis}

All quantified data represent the mean \pm SEM. Statistical significance was assessed by the $t$ test and one- or two-way analysis of variance (ANOVA), and a Bonferroni post-hoc test was used to test multiple comparisons. All statistical analyses were performed with SPSS (version 16.0; SPSS, Chicago, IL, USA) and significance was defined as $P<0.05$.

\section{Results}

The influence of $\mathrm{OGD} / \mathrm{R}$ on the temporal expression pattern of Tie2 and the activation of downstream signaling molecules in BECs

In a recent study, we demonstrated that $\mathrm{OGD} / \mathrm{R}$ induced a markedly increased expression of both integrin $\alpha 5$ and Ang1 on BECs, with maximum levels of expression reached $48-72 \mathrm{~h}$ post-restoration ${ }^{9}$. In the current study, we wanted to evaluate whether OGD/R also influenced BEC expression of the Ang1 receptor Tie2. To examine this, we subjected bEnd3 cells and HBMECs to $4 \mathrm{~h}$ of OGD, followed by up to $72 \mathrm{~h}$ of restoration (normal glucose and normoxia). We then examined total protein, the phosphorylated form of Tie2, and the expression of integrin $\alpha 5$ and Ang1 by western blotting.

As shown in Fig. 1a, b, western blot analysis revealed that compared to control conditions, the levels of total 


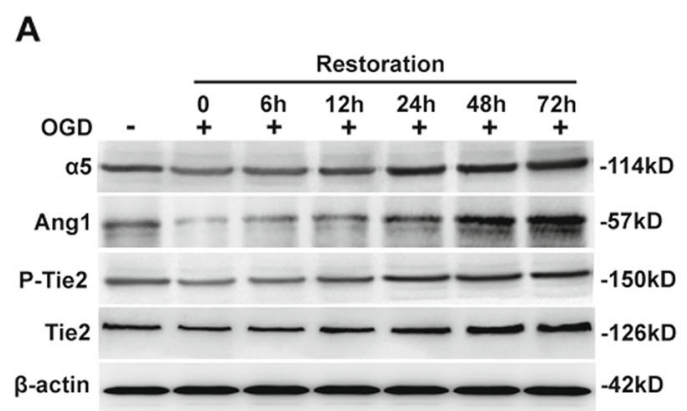

C

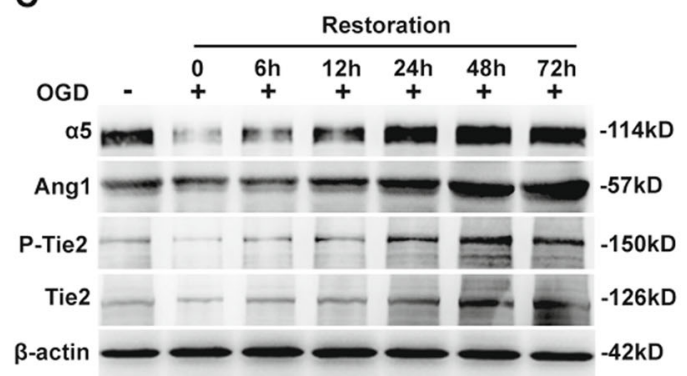

E

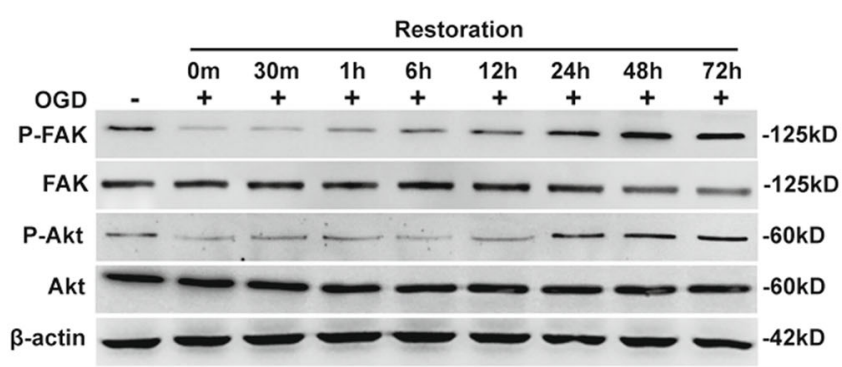

B

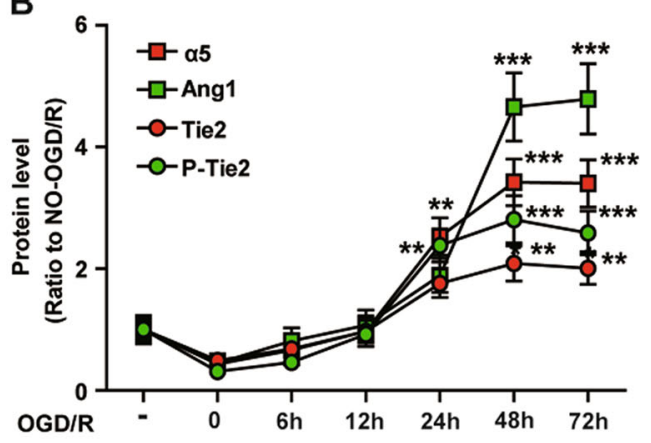

D

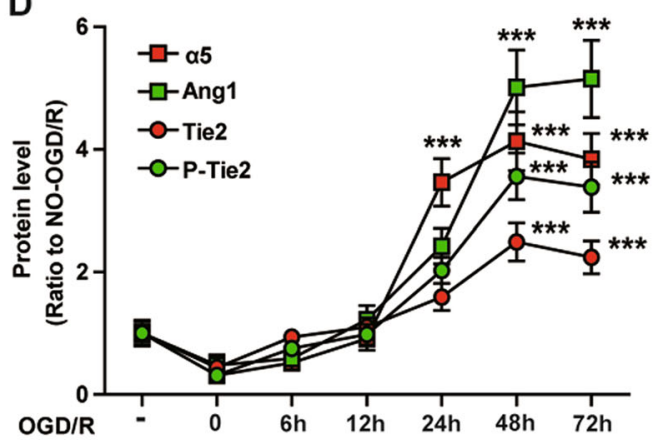

$\mathbf{F}$

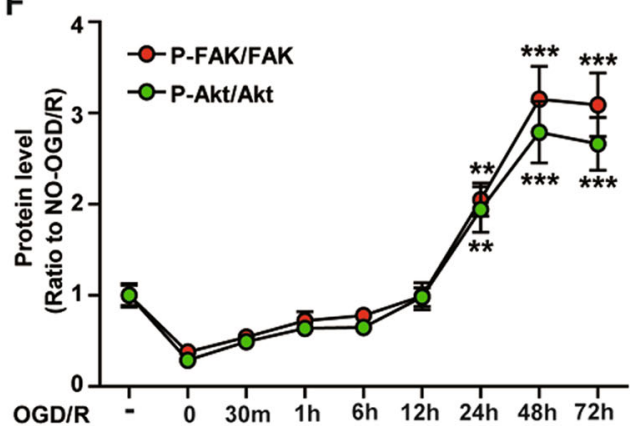

Fig. $1 \mathrm{OGD} / \mathrm{R}$ induces the upregulation of Tie2 expression and activation of the downstream signaling molecules FAK and Akt in BECs. BECs (bEnd3 cells and HBMECs) were subjected to $4 \mathrm{~h}$ of oxygen-glucose deprivation (OGD) followed by $72 \mathrm{~h}$ of restoration (R) at $37^{\circ} \mathrm{C}$. Cell lysates at each time point were analyzed by western blotting using antibodies specific for integrin a5, Ang1, and total protein or phosphorylated form of Tie2, focal adhesion kinase (FAK) and Akt. a Representative western blots of integrin a5, Ang 1 and total and phosphorylated form of Tie2 in bEnd3 cells are shown. $\mathbf{b}$ Protein levels for $\mathbf{A}$ were quantified by densitometry and are presented as ratios to $\beta$-actin. $\mathbf{c}$ Representative western blots of integrin a5, Ang 1 and total and phosphorylated form of Tie2 in HBMECs are shown. $\mathbf{d}$ Protein levels for $\mathbf{C}$ were quantified by densitometry and are presented as ratios to $\beta$-actin. e Representative western blots of phosphorylated FAK, phosphorylated Akt, total FAK and total Akt are shown. $\mathbf{f}$ Protein levels were quantified by densitometry and normalized to total FAK/Akt. The NO-OGD/R cells served as a control. Note that the levels of phosphorylated and total Tie2 decreased immediately after OGD insult but then began to increase and continued to increase with time of restoration, reaching a maximum at 48-72 $\mathrm{h}$ after restoration, similar to the expression of a5 integrin and Ang1. In the first $12 \mathrm{~h}$ following ischemic insult, OGD/R reduced phosphorylation of both FAK and Akt, but by the $24-72 \mathrm{~h}$ time point, the levels of both phosphorylated FAK and Akt were significantly elevated compared to pre-OGD conditions, which correlated with the changes of total and phosphorylated form of Tie2. Data represent the mean \pm SEM and were analyzed by one-way ANOVA $(\boldsymbol{n}=5)$. ${ }^{* *} P<0.01,{ }^{* * *} P<0.001$ compared with NO-OGD/R control

and phosphorylated Tie2 decreased immediately after OGD insult in bEnd3 cells, but then began and continued to increase with time of restoration to reach a maximum between 48 and $72 \mathrm{~h}$ after restoration. BEC expression of a5 integrin and Ang1 (Fig. 1a, b, additional Fig. 1) showed a similar response.
Quantification revealed that compared to NO-OGD/R control conditions, the expression of Tie 2 was increased $2.38 \pm 0.27$-fold for phosphorylated Tie2 $(P<0.01)$ and $1.76 \pm 0.23$-fold for total Tie2 $(P>0.05)$ at $24 \mathrm{~h}$, further increased $2.81 \pm 0.39$-fold for phosphorylated Tie2 $(P<$ $0.001)$ and $2.09 \pm 0.29$-fold for total Tie $2(P<0.01)$ at $48 \mathrm{~h}$, 

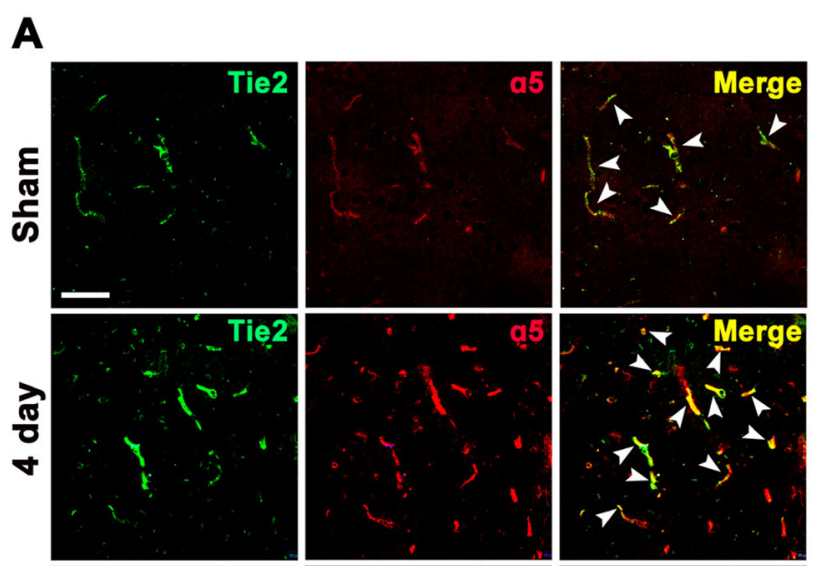

\section{B}
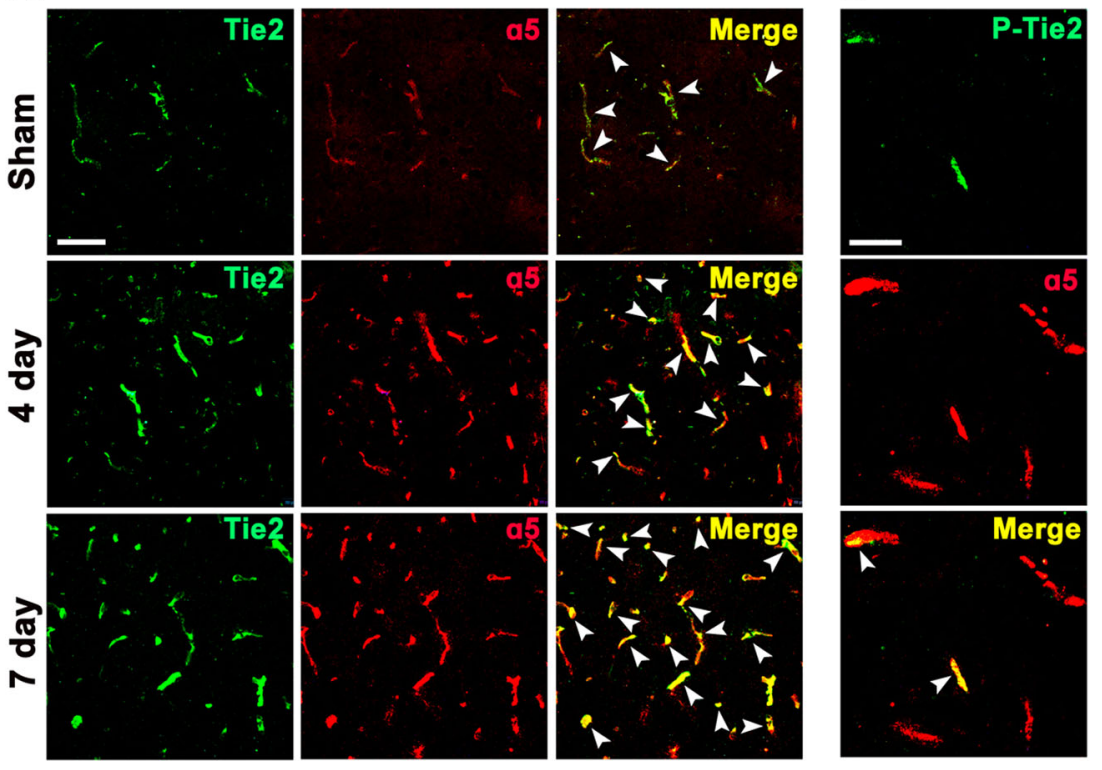

Fig. 2 Coexpression of Tie2/P-Tie2 with a5 integrin on cerebral vessels after cerebral ischemia. Dual-IF was performed on frozen sections of ischemic penumbra taken from sham-operated (control) mice at 4 and 7 days after focal cerebral ischemia, using antibodies specific for a5 (Cy-3), Tie2 (AlexaFluor-488) (a)/a5 (Cy-3), or P-Tie2 (AlexaFluor-488) (b). Scale bar $=50 \mathrm{~mm}$ for all panels in figure A, Scale bar $=30 \mathrm{~mm}$ for all panels in figure B. Note that cerebral ischemia induced a strong increase in vascular expression of Tie2 and a 5 integrin. In the ischemic penumbra, Tie2 and a5 integrin co-localized (white arrows) extensively. In contrast, only relatively low levels of Tie2 co-localize with a 5 integrin (white arrows) in the control (sham) brain. The activated Tie2 (P-Tie2) co-localized with a5 integrin (white arrows) in the ischemic penumbra

and increased $2.59 \pm 0.36$-fold for phosphorylated Tie2 $(P<0.001)$ and $2.01 \pm 0.27$-fold for total Tie2 $(P<0.01)$ at $72 \mathrm{~h}$. Similarly, the expression of $\alpha 5$ integrin was increased $2.53 \pm 0.31$-fold $(P<0.01)$ at $24 \mathrm{~h}$, further increased $3.42 \pm$ 0.38 -fold $(P<0.001)$ at $48 \mathrm{~h}$ and increased $3.4 \pm 0.39$-fold $(P<0.001)$ at $72 \mathrm{~h}$. In parallel, the expression levels of Ang1 were increased $1.89 \pm 0.28$-fold $(P>0.05)$ at $24 \mathrm{~h}$, significantly increased $4.66 \pm 0.56$-fold $(P<0.001)$ at $48 \mathrm{~h}$, and further increased $4.79 \pm 0.58$-fold $(P<0.001)$ at $72 \mathrm{~h}$ (Fig. 1b).

In addition, we used HBMECs to repeat the above studies and found that integrin $\alpha 5$, Ang1, and the total and phosphorylated form of Tie2 in HBMECs changed in similar manners (Fig. 1c, d).

Autophosphorylation of Tie2 triggered by Ang1 binding has been reported to activate specific intracellular signal molecules, including FAK, Akt, and mitogen-activated protein kinase (MAPK) (ERK1/2) in endothelial cells, which have been shown to selectively regulate vascular quiescence and angiogenesis ${ }^{12,14}$. Considering this, we next examined how OGD/R affects phosphorylation of the Tie2 receptor downstream signaling molecules FAK and Akt on BECs.

As expected, in the first $12 \mathrm{~h}$ following the ischemic insult, $\mathrm{OGD} / \mathrm{R}$ induced a reduction in phosphorylation of both FAK and Akt in BECs, but by the 24-48-h time point, the endothelial levels of both phosphorylated FAK and Akt were significantly elevated compared to pre-OGD conditions (Fig. 1e, f), and this was associated with enhanced expression of phosphorylated and total Tie2. Quantification revealed that compared to the NO-OGD/R control conditions, phosphorylation of FAK was increased to $2.05 \pm 0.18$-fold $(P<0.01)$ at $24 \mathrm{~h}$, further increased to $3.15 \pm 0.36$-fold $(P<0.001)$ at $48 \mathrm{~h}$, and increased to $3.09 \pm 0.35$-fold $(P<0.001)$ at $72 \mathrm{~h}$, and the phosphorylation level of Akt was increased to $1.94 \pm 0.25$-fold $(P<$ $0.01)$ at $24 \mathrm{~h}$, further increased to $2.79 \pm 0.34$-fold $(P<$ $0.001)$ at $48 \mathrm{~h}$, and increased to $2.66 \pm 0.29$-fold $(P<0.001)$ at $72 \mathrm{~h}$ (Fig. 1f).

\section{$\mathrm{OGD} / \mathrm{R}$-induced Tie2 receptor activation on BECs is mediated by $a 5 \beta 1$ integrin}

As Tie2 has been shown to interact selectively and constitutively with $\alpha 5 \beta 1$ integrin $^{10}$, we next performed dual-IF to examine whether Tie2/P-Tie 2 and $\alpha 5$ integrin subunits show any overlap in their expression profiles after cerebral ischemia. As shown in Fig. 2a, cerebral ischemia induced a strong increase in vascular expression of Tie 2 and $\alpha 5$ integrin at days 4 and 7 post ischemia compared with the control (sham) brain. Integrin $\alpha 5 \beta 1$ extensively co-localized with Tie 2 on cerebral vessels in the ischemic penumbra at 4 and 7 days post ischemia. In addition, the coexpression of P-Tie 2 with $\alpha 5$ integrin can be observed on cerebral vessels in the ischemic penumbra 


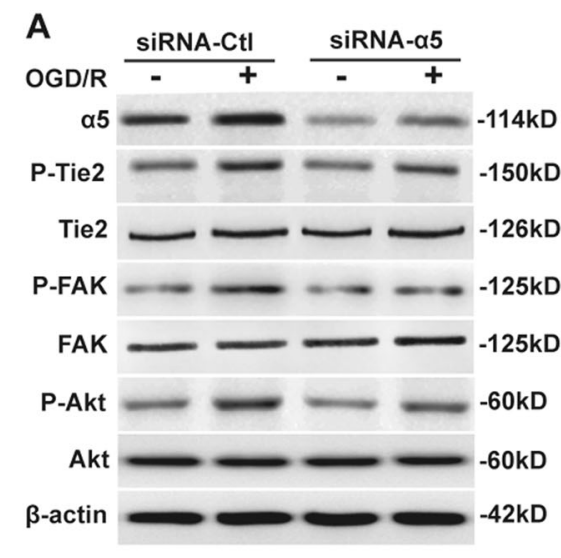

B

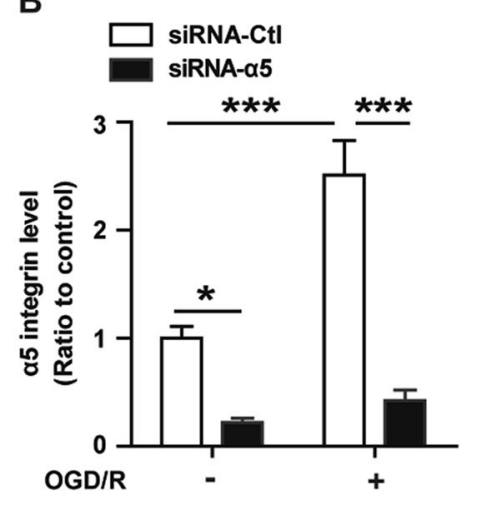

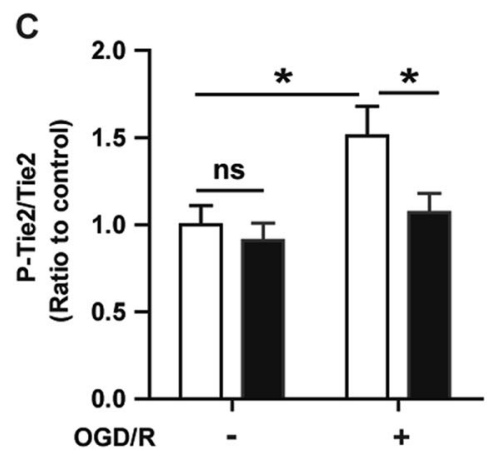

D

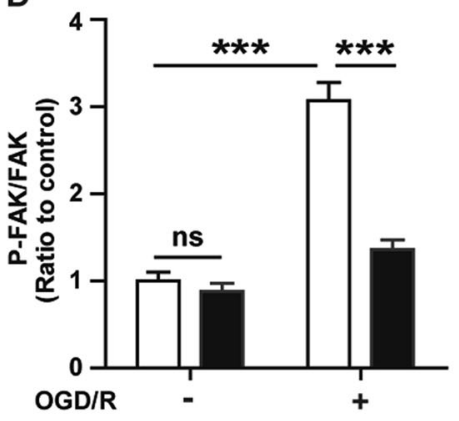

E

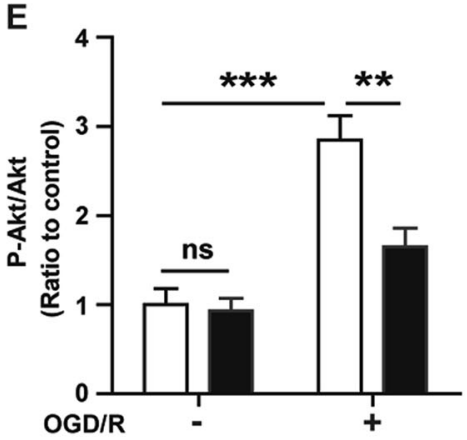

Fig. 3 Knockdown of a5 integrin attenuates Tie2 activation in BECs induced by OGD/R. BECs were transfected with the negative control siRNA (siRNA-Ctl) or a5 integrin-specific siRNA (siRNA-a5) for $48 \mathrm{~h}$ and then subjected to either $4 \mathrm{~h}$ of OGD followed by $48 \mathrm{~h}$ of restoration or NO-OGD/R at $37^{\circ} \mathrm{C}$. a Cell lysate protein levels were analyzed by western blotting using antibodies specific to integrin a 5 and total or phosphorylated forms of Tie2, FAK, and Akt. b-e. Bar graphs show the quantitative analyses of western blots as ratios of integrin a5/ $\beta$-actin (b), phosphorylated Tie2/total Tie2 (c), phosphorylated FAK/total FAK (d), and phosphorylated Akt/total Akt (e). The NO-OGD/R siRNA-Ctl-treated cells served as the control. Data represent the mean \pm SEM and were analyzed by two-way ANOVA $(\boldsymbol{n}=\mathbf{5})$. Note that OGD/R significantly increased the levels of Tie2, FAK, and Akt phosphorylation on siRNA-Ctl-treated BECs relative to the NO-OGD/R siRNA-Ctl-treated controls, but this effect was significantly reduced by diminishing the levels of integrin a5 in the BECs. ${ }^{*} P<0.05$, ${ }^{*} P<0.01$, ${ }^{* *} P<0.001$; ns, not significant

4 days post ischemia (Fig. 2b). These results show that there is a direct interaction between $\alpha 5 \beta 1$ integrin and Tie2, and that $\alpha 5 \beta 1$ integrin might be involved in the activation of Tie2 after cerebral ischemia.

To confirm if $\alpha 5 \beta 1$ integrin mediates OGD/R-induced Tie2 activation in BECs, we employed $\alpha 5$ integrin-specific siRNA to knockdown the expression of $\alpha 5$ integrin in BECs and examined the impact of this on Tie2 receptor signaling activation in response to $\mathrm{OGD} / \mathrm{R}$. As shown in Fig. $3 \mathrm{a}-\mathrm{e}, 48 \mathrm{~h}$ after restoration, expression of phosphorylated Tie 2 and its downstream effectors FAK and Akt in the negative control siRNA (siRNA-Ctl)-treated BECs was significantly induced in response to OGD/R relative to the NO-OGD/R siRNA-Ctl-treated cells (the controls) ( $P<0.05$ or $\left.{ }^{* * * *} P<0.001\right)$, but this effect was markedly attenuated following integrin $\alpha 5$ knockdown. These data suggest that integrin $\alpha 5 \beta 1$ regulates the OGD/ $\mathrm{R}$-induced Tie 2 receptor activation on BECs.

To investigate whether $\alpha 5 \beta 1$ integrin directly induces Tie2 signaling, BECs were transfected with negative control siRNA (siRNA-Ctl) or $\alpha 5$ integrin-specific siRNA (siRNA- $\alpha 5$ ) for $48 \mathrm{~h}$, then subjected to $4 \mathrm{~h}$ of OGD, and further stimulated or not with Ang1 (50, 100, or $200 \mathrm{ng} /$ $\mathrm{mL}$ ) for $15 \mathrm{~min}$ under normoxia at $37^{\circ} \mathrm{C}$. As shown in Fig. 4a-d, knockdown of integrin $\alpha 5$ in BECs did not significantly decrease the levels of Tie2 phosphorylation after OGD (Fig. 4b, d). However, Ang1 (at doses of 50, 100 and $200 \mathrm{ng} / \mathrm{mL}$ ) significantly increased the levels of Tie2 phosphorylation in a dose-dependent manner on siRNACtl-treated BECs after OGD compared with the nonAng1 treatment group $\left({ }^{*} P<0.05\right.$ or $\left.{ }^{* * *} P<0.001\right)$, but this effect was significantly reduced by integrin $\alpha 5$ knockdown (Fig. 4b, d). These data suggest that $\alpha 5 \beta 1$ integrin does not directly induce Tie2 signaling, but acts to enhance Ang-1-activated Tie2 signaling on BECs after OGD.

\section{Priming of BECs with Ang1 promotes the expression of a5 integrin and Ets-1 after OGD}

In our recent study, we demonstrated extensive colocalization of Ang1 with $\alpha 5$ integrin on angiogenic blood 


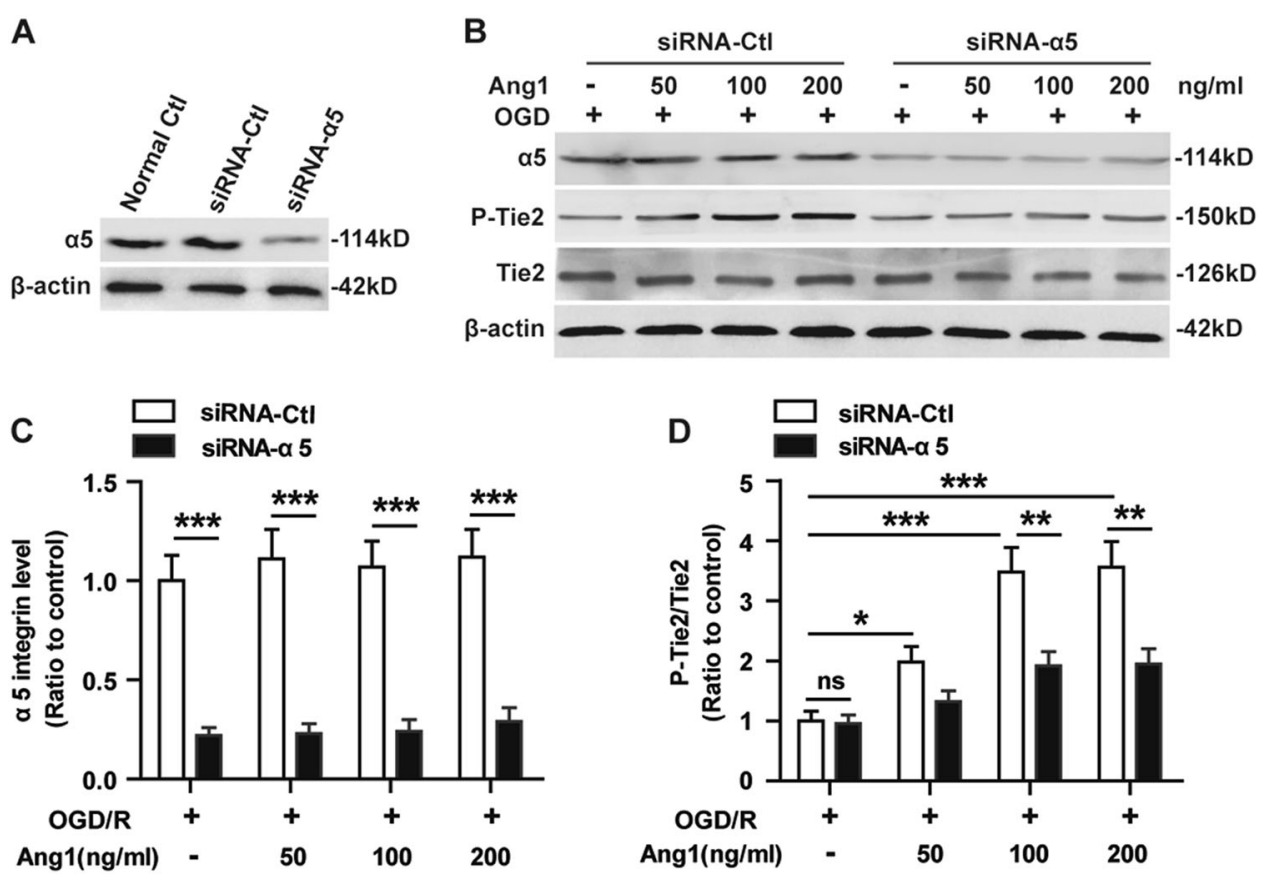

Fig. 4 Integrin $\mathbf{a} 5 \beta 1$ acts to enhance Ang1-activated Tie2 signaling after OGD. a BECs were transfected with negative control siRNA (siRNA-Ctl) or a5 integrin-specific siRNA (siRNA-a5) for $48 \mathrm{~h}$. Levels of a5 $\beta 1$ protein were monitored in the control and silenced cells via western blotting. b-d BECs were transfected with siRNA-Ctl or siRNA-a5 for $48 \mathrm{~h}$, then subjected to $4 \mathrm{~h}$ of OGD, and further stimulated or not with Ang 1 (50, 100, or $200 \mathrm{ng} / \mathrm{mL}$ ) for $15 \mathrm{~min}$ under normoxia at $37^{\circ} \mathrm{C}$. b The protein levels from OGD-treated BEC lysates were analyzed by western blotting using antibodies specific to integrin a5 and total or phosphorylated forms of Tie2. c-d Densitometric analysis shows the relative amount of integrin a5 (c) and phosphorylated Tie2/total Tie2 (d). The OGD/R siRNA-Ctl-treated cells served as the control. Data represent the mean \pm SEM and were analyzed by two-way ANOVA $(n=5)$. Note that integrin a5 knockdown in BECs significantly decreased a5 $\beta 1$ protein expression after OGD. Knockdown of integrin a5 in BECs did not significantly decrease the levels of Tie2 phosphorylation after OGD. Ang1 significantly increased the levels of Tie2 phosphorylation in a dose-dependent manner on siRNA-Ctl-treated BECs after OGD, but this effect was significantly reduced by integrin a5 knockdown. ${ }^{*} P<0.05$, ${ }^{* * *} P<0.001$, ns, not significant

vessels in the ischemic penumbra, suggesting that an interaction between Ang1 and a5 integrin might be important in promoting angiogenesis after ischemic stroke ${ }^{9}$. A previous report showed that short-term stimulation of peripheral blood stem cells enriched by granulocyte colony-stimulating factor mobilization and apheresis (mobPBSCs) with COMP-Ang1 induced the expression of $\alpha 5 \beta 1$ integrin through Tie2 receptor downstream molecule Ets-1 signaling ${ }^{11}$. To examine whether a similar regulation exists in BECs under OGD conditions, we stimulated BECs with Ang1 (100 ng/mL) for 1 or $12 \mathrm{~h}$ immediately after OGD insult and then examined the protein levels of $\alpha 5$ integrin and Ets- 1 by western blotting. As shown in Fig. 5a-c, stimulation of OGD-treated BECs with Ang1 for $1 \mathrm{~h}$ did not change the expression levels of $\alpha 5$ integrin or Ets-1; however, stimulation of OGD-treated BECs with Ang1 for $12 \mathrm{~h}$ significantly increased the expression of both $\alpha 5$ integrin and Ets-1 compared with the non-Ang1 treatment group (1.77 \pm 0.21 -fold versus $1.05 \pm 0.14$-fold for $\alpha 5$ integrin, $P<0.05 ; 2.61 \pm 0.39$-fold versus $0.97 \pm 0.19$-fold for Ets- 1 , $P<0.01$ ) (Fig. 5b, c). These results indicate that priming of
BECs with Ang1 promotes the expression of integrin $\alpha 5$ and Ets-1 under OGD/R conditions.

\section{Ets-1 is necessary for the enhanced expression of a5 integrin induced by Ang1 after OGD/R}

Next, we examined whether the enhanced expression of $\alpha 5$ integrin induced by Ang 1 after OGD/R is mediated by the regulation of Ets-1. BECs were transfected with the negative control siRNA (siRNA-Ctl) or Ets-1-specific siRNA (siRNA-Ets-1) for $48 \mathrm{~h}$, then subjected to $4 \mathrm{~h}$ of OGD, and further stimulated or not with Ang1 (100 ng/ $\mathrm{mL}$ ) for $12 \mathrm{~h}$ under normoxia at $37^{\circ} \mathrm{C}$. As shown in Fig. 6, Ang1 significantly induced the expression of integrin $\alpha 5$ on siRNA-Ctl-treated BECs relative to the OGD/R siRNA-Ctl-treated controls $\left({ }^{*} P<0.05\right)$. Furthermore, knockdown of Ets-1 expression in BECs markedly decreased integrin $\alpha 5$ expression in the presence or absence of Ang1 after OGD (" $P<0.05$ or ${ }^{* * *} P<0.001$ ) (Fig. 6a-d). To directly investigate the effect of knockdown of Ets- 1 on the upregulation of $\alpha 5$ integrin induced by Ang 1 after $\mathrm{OGD} / \mathrm{R}$, we compared the difference between the fold increases of integrin $\alpha 5$ induced by Ang 1 

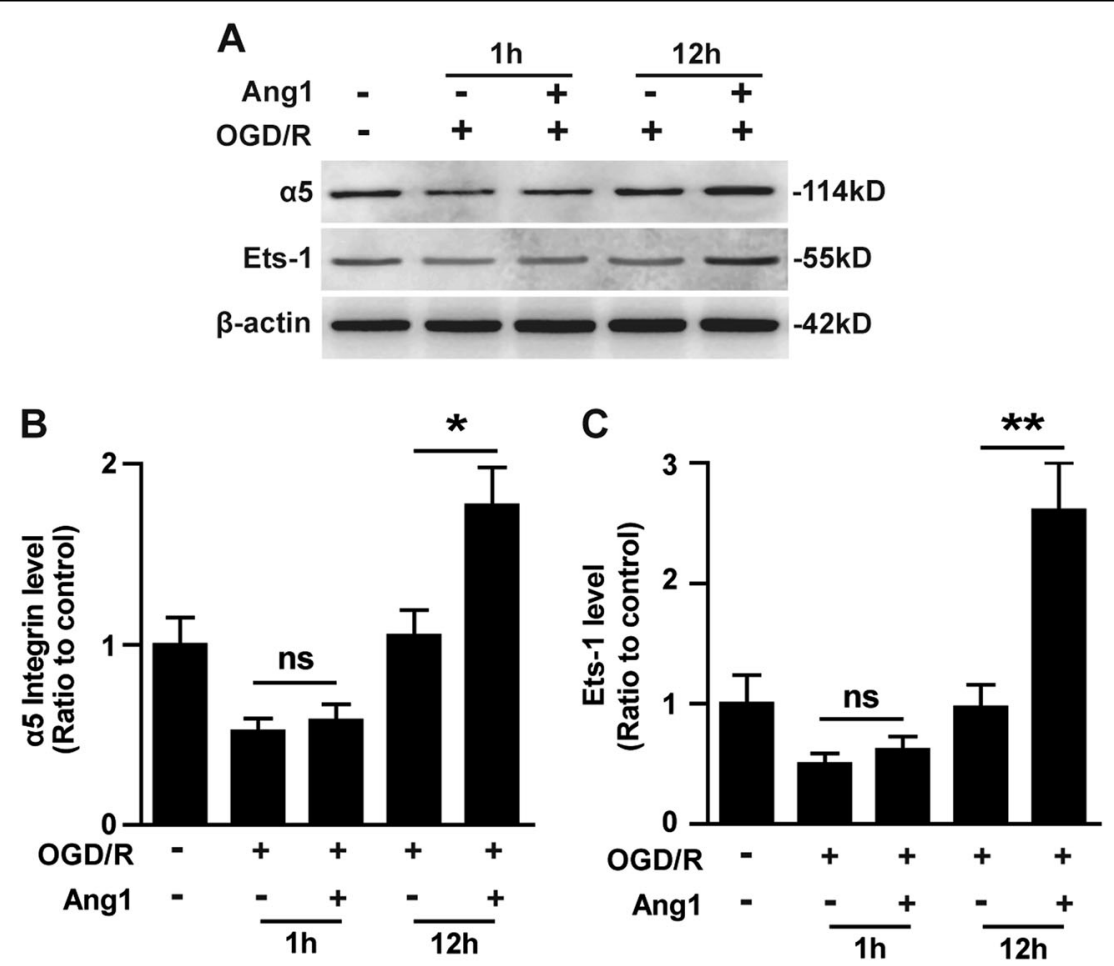

Fig. 5 Ang1 promotes BEC expression of integrin a5 and Ets-1 under OGD/R conditions. BECs were subjected to $4 \mathrm{~h}$ of $\mathrm{OGD}$ and then stimulated or not with $100 \mathrm{ng} / \mathrm{mL}$ of Ang1 for the indicated periods under normoxia at $37^{\circ} \mathrm{C}$. a Cell lysates from BECs were analyzed to assess the levels of the indicated proteins by western blotting. $\mathbf{b}$, c Protein levels of integrin a5 (b) and Ets-1 (c) were quantified by densitometry and are presented as ratios to $\beta$-actin. The NO-OGD cells served as the control. Data represent the mean \pm SEM and were analyzed by one-way ANOVA ( $n=$ 3). Note that stimulation of OGD-treated BECs with Ang1 for $12 \mathrm{~h}$ significantly increased the expressions of both a5 and Ets-1.*P<0.05, ${ }^{* *} P<0.01$; ns, not significant

in siRNA-Ctl-treated controls and siRNA-Ets-1-treated BECs after OGD. This comparison revealed that diminishing the levels of Ets-1 in BECs significantly reduced the increase rate of integrin $\alpha 5$ induced by Ang1 after OGD compared to the siRNA-Ctl-treated controls $(* P<0.05)$ (Fig. 6e). These results indicate that the enhanced expression of $\alpha 5$ integrin induced by Ang 1 after OGD/R is dependent on the upregulation of Ets-1.

\section{Ang1-induced BEC migration and tube formation after OGD is mediated by integrin a5}

Because endothelial cell migration and tube formation constitute an important process in blood vessel formation and both the Ang1-Tie2 system ${ }^{15}$ and integrin $\alpha 5 \beta 1^{16}$ are key regulators of the angiogenic process, we tested whether integrin $\alpha 5 \beta 1$-Ang1/Tie2 receptor cross-talk regulates the migration and tube formation of BECs.

BECs were transfected with either siRNA-Ctl or $\alpha 5$ integrin-specific siRNA (siRNA- $\alpha 5$ ), and then, cell migration and tube formation were measured after transfected BECs were subjected to $4 \mathrm{~h}$ of OGD. As illustrated in Fig. 7a, b, Ang1 treatment significantly enhanced BEC migration after $24 \mathrm{~h}$ of wound scratch healing relative to the siRNA-Ctl-treated controls $(P<$ 0.001 ), but this increase was attenuated by diminishing the levels of $\alpha 5$ integrin in the BECs. Similarly, as demonstrated in Fig. 7c, d, considerable tube formation occurred when BECs were incubated on Matrigel for $12 \mathrm{~h}$, and Ang1 strongly stimulated the BEC tube formation $(P<0.001)$ vs. the control. However, the Ang1-induced capillary tube formation was inhibited by diminishing the levels of $\alpha 5$ integrin in the BECs. These results indicate that the Ang1-induced migration and tube formation of BECs after OGD is mediated by integrin $\alpha 5$.

\section{Discussion}

In the current study, we demonstrate for the first time that the Ang1/Tie2 system cross-talks with integrin $\alpha 5 \beta 1$ on BECs after experimental in vitro cerebral ischemia. Our main findings were as follows: (i) BECs subject to OGD/R showed increased expression and activation of Tie2, (ii) induction of Tie2 receptor activation by OGD/R is mediated by $\alpha 5 \beta 1$ integrin, (iii) priming of BECs with Ang1 promotes the expression of $\alpha 5$ integrin and Ets- 1 after OGD, (iv) Ets-1 is necessary for Ang1 induction of $\alpha 5$ integrin expression on BECs after OGD/R, and 

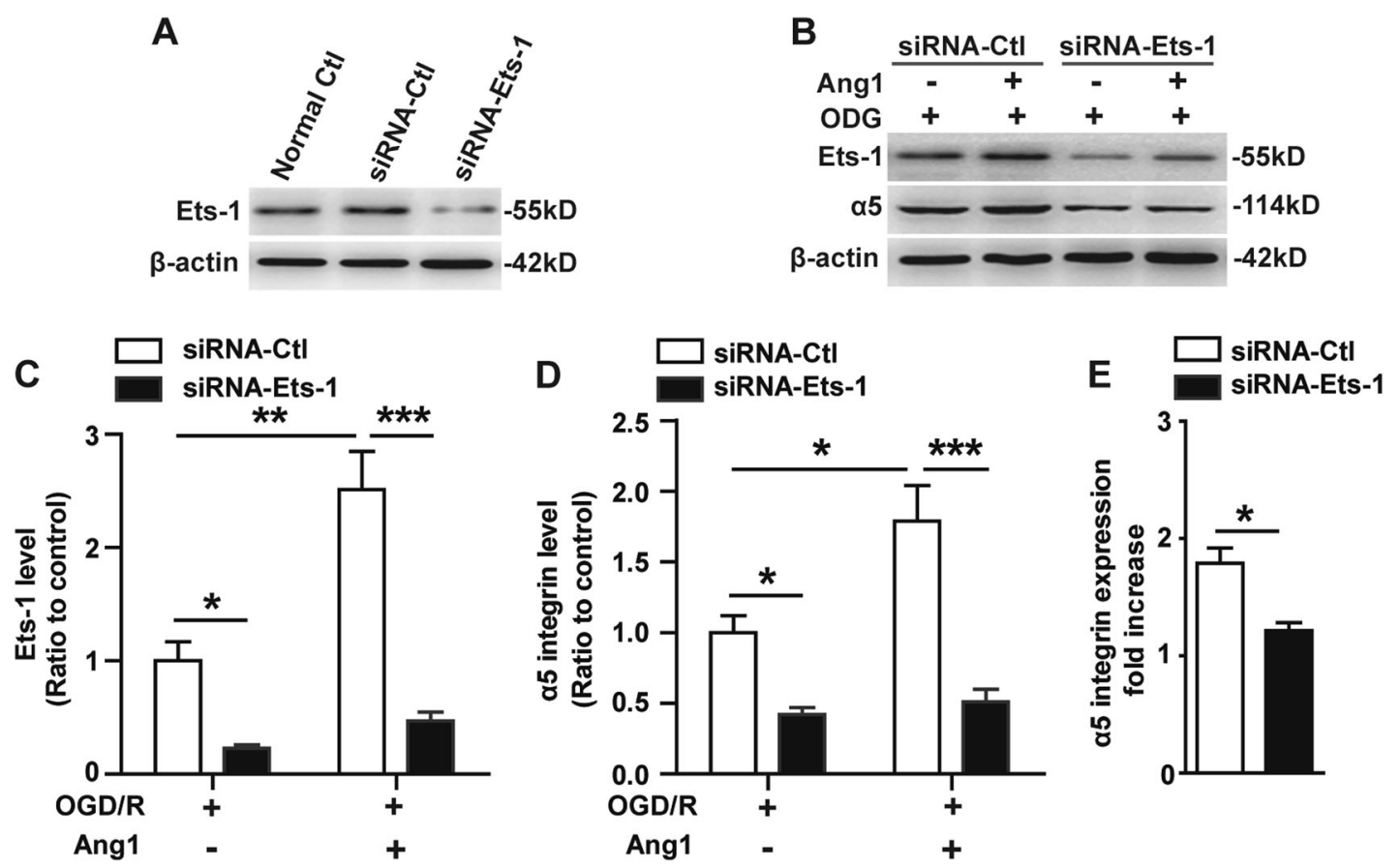

Fig. 6 Knockdown of Ets-1 reverses the upregulation of a5 integrin induced by Ang1 after OGD. a. BECs were transfected with the negative control siRNA (siRNA-Ctl) or Ets-1-specific siRNA (siRNA-Ets-1) for $48 \mathrm{~h}$. Levels of Ets-1 protein were monitored in the control and silenced the cells via western blotting. b-e BECs were transfected with siRNA-Ctl or siRNA-Ets-1 for $48 \mathrm{~h}$, then subjected to $4 \mathrm{~h}$ of OGD, and further stimulated or not with Ang1 $(100 \mathrm{ng} / \mathrm{mL})$ for $12 \mathrm{~h}$ under normoxia at $37^{\circ} \mathrm{C}$. $\mathbf{b}$ The protein levels from OGD-treated BECs lysates were analyzed by western blotting using antibodies specific to integrin a5 and Ets-1. c-e Densitometric analysis shows the relative amount of Ets-1 (c) and integrin a5 (d) to $\beta$-actin (the OGD/ R siRNA-Ctl-treated cells served as the control) and the fold increase of integrin a5 induced by Ang1 after OGD (e). Data represent the mean \pm SEM and were analyzed by two-way ANOVA or Student's $t$ test $(n=3)$. Note that Ets-1 knockdown in BECs significantly decreased Ets-1 protein expression. Ang1 significantly upregulated integrin a5 expression on siRNA-Ctl-treated BECs relative to the OGD/R siRNA-Ctl-treated controls, but this effect was significantly reduced by Ets-1 knockdown. ${ }^{*} P<0.05,{ }^{* *} P<0.01,{ }^{* *} P<0.001$

(v) Ang1-induced migration and tube formation of BECs after OGD is mediated by integrin $\alpha 5$.

\section{Regulation of Ang1/Tie2 receptor signaling in BECs after OGD/R}

Our recent study showed that vascular expression of both integrin $\alpha 5$ and Ang1 decreased during the early stages of vascular remodeling, but then increased in the later stages under cerebral ischemic conditions in vivo and in vitro ${ }^{1,9}$. The induction of $\alpha 5 \beta 1$ was first noticeable after 4 days post ischemia in vivo, followed by an increase in Ang1 at day 7 post ischemia ${ }^{1,9}$, which is consistent with the time course of the response to OGD/R at 24 and $48 \mathrm{~h}$ for $\alpha 5 \beta 1$ and Ang1 on BECs in vitro, respectively, indicating that the induction of Ang1 lags behind that of $\alpha 5 \beta 1$ after cerebral ischemia. In the current study, we found that OGD/R induced the expression of the Ang1 receptor Tie2 on BECs in a manner similar to that for integrin $\alpha 5$ and Ang1 in response to OGD/R, which is consistent with previous reports ${ }^{17}$ and our current study showing increased Tie2 expression in the ischemic border area in vivo.
We also noted that upregulation of Tie2 was accompanied by increased phosphorylation (activation) of this receptor as well as increased phosphorylation of FAK and Akt in response to OGD/R. These observations strongly suggest that $\mathrm{OGD} / \mathrm{R}$ induces Ang1/Tie2 receptor signaling in BECs. As Ang1-Tie2 signaling is a key regulator of angiogenesis and adult vascular homeostasis ${ }^{15}$, it seems likely that enhanced Ang1/Tie2 receptor signaling will promote endogenous angiogenesis and vascular protection after cerebral ischemia.

\section{Integrin $a 5 \beta 1$ regulation of cerebral ischemia-induced activation of Tie2 in BEC}

In the current study, we found that $\alpha 5 \beta 1$ integrin colocalized with Tie2/P-Tie2 on the cerebral vessels in the ischemic penumbra after focal cerebral ischemia, showing that there is a direct interaction between $\alpha 5 \beta 1$ integrin and Tie2, and that $\alpha 5 \beta 1$ integrin might be related to the activation of Tie 2 after cerebral ischemia. To confirm this, we next investigated the effect of integrin $\alpha 5 \beta 1$ on the activation of Tie2 in response to OGD/R. Our gene silencing experiment confirmed that diminishing $\mathrm{BEC}$ integrin $\alpha 5 \beta 1$ 

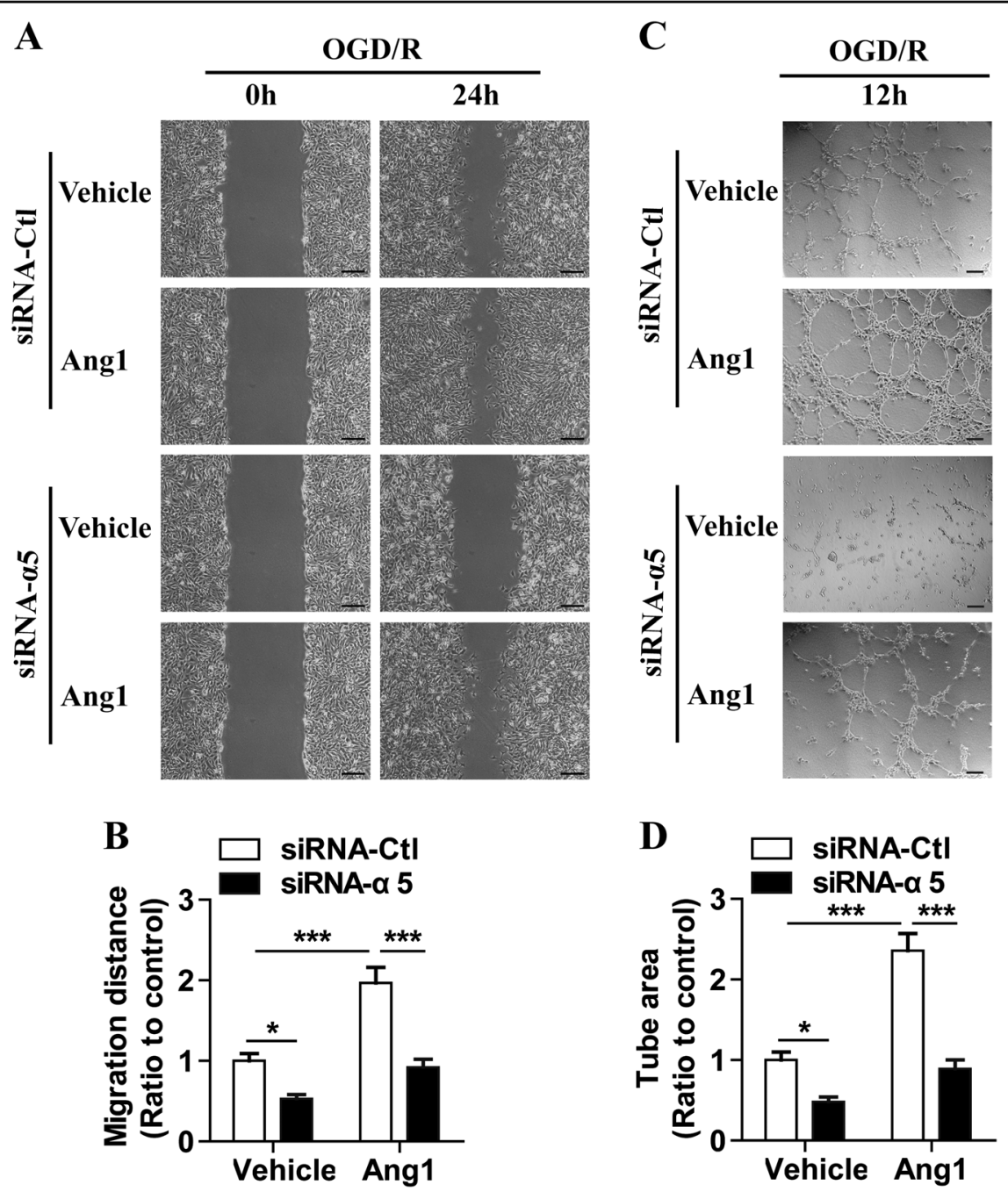

Fig. 7 Ang1-induced BEC migration and tube formation after OGD is mediated by integrin a5. BECs were transfected with siRNA-Ctl or a5 integrin-specific siRNA (siRNA-a5), and then, cell migration and tube formation were measured after transfected BECs were subjected to $4 \mathrm{~h}$ of OGD. a Representative images of cell migration in monolayer cultured BECs in the absence or presence of Ang $1(100 \mathrm{ng} / \mathrm{mL})$ as indicated, scale bar $=$ $200 \mu \mathrm{m}$. b Quantification of BEC cell migration. The relative migration distance was quantified using ImageJ software and expressed as the ratio to the values of the siRNA-CtI-treated cells (control). c Representative images of capillary tube formation of BECs on Matrigel-coated plates in the absence or presence of Ang $1(100 \mathrm{ng} / \mathrm{mL})$ as indicated, scale bar $=100 \mu \mathrm{m}$. d Quantification of tube formation. Data represent the mean \pm SEM and were analyzed by two-way ANOVA $(n=3)$. Note that Ang1 significantly increased the migration and capillary-like tube formation of BECs after OGD, but this effect was significantly inhibited by diminishing the levels of a5 in BECs. ${ }^{*} P<0.05,{ }^{* *} P<0.001$

expression via selective shRNA- $\alpha 5$ resulted in the attenuation of OGD/R-induced phosphorylation of Tie2, FAK, and Akt in BECs, but the overall protein levels of Tie2 remained unaffected. The in vivo and in vitro findings suggest that integrin $\alpha 5 \beta 1$ is involved in the regulation of Tie2 receptor activation in BECs after cerebral ischemia. $\beta 1$ integrin has previously been shown to induce phosphorylation of FAK and Akt following ligation with collagen ${ }^{18}$ and may thus influence the levels independently of Ang1/Tie2. However, in the current study, knockdown of $\alpha 5 \beta 1$ integrin in BECs did not change the phosphorylation levels of Tie2, FAK, and Akt in BECs under normal conditions. As phosphorylation of Tie 2 triggered by Ang1 binding has been reported to activate specific intracellular signal molecules, including FAK, Akt, and mitogen-activated protein kinase (MAPK) (ERK1/2), in endothelial cells ${ }^{12,14}$, it seems that $\alpha 5 \beta 1$ integrin interaction with Ang1/Tie2 enhanced the activation of Tie2 and its downstream effectors FAK and Akt in BECs after cerebral ischemia. This is further confirmed by the dose response to Ang1 in both shRNA- $\alpha 5$ and siRNACtl-treated OGD/R cells and is consistent with experiments showing that $\alpha 5 \beta 1$ integrin does not directly induce Tie2 signaling, but acts to sensitize Ang1-induced Tie2 activation after cerebral ischemia. 
In an alternative signaling pathway, Dalton et $\mathrm{al}^{14}$ demonstrated that the levels of activated Akt increase in response to the Tie2 ligand Ang1, but the elevated levels of phosphorylated Akt induced by Ang1 were not significantly affected in $\alpha 5$ integrin knockdown telomeraseimmortalized endothelial cells (TIMEs). The mechanism behind these apparent differences, from our results, may relate to the different experimental conditions, including sources of endothelial cells (Dalton used TIMEs, while our studies employed BECs). In addition, in our model, BECs were subjected to OGD/R treatment, while in the Dalton study, TIMEs were stimulated by Ang1 under normal (non-OGD) conditions. Another reason may be related to the different harvesting time points, as we examined the effect of integrin $\alpha 5$ knockdown on the activation of Tie2 and its downstream effectors at $48 \mathrm{~h}$ post-restoration, while the Dalton study monitored the changes of phosphorylated Akt just 15 min after Ang1 stimulation.

\section{Ang1 induction of a5 integrin expression on BECs through Ets-1 signaling after OGD}

Another novel finding of our study was that priming BECs with Ang1 for $12 \mathrm{~h}$ promoted the expression of $\alpha 5$ integrin after OGD, although this phenomenon was not observed when BECs were stimulated with Ang1 for only $1 \mathrm{~h}$ immediately after OGD insult, presumably because it takes this long for $\alpha 5$ integrin protein to be upregulated at the cell surface. We also found that priming of BECs with Ang1 for $12 \mathrm{~h}$ induced the upregulation of Ets-1. Ets-1, a member of the v-ets erythroblastosis virus E26 oncogene homolog (ETS) transcription factors, is known to be expressed in endothelial cells during angiogenesis ${ }^{19}$ and be involved in the upregulation of hypoxic-inducible genes $^{20}$. Importantly, Tie2 is reported to contain Ets-1 transcription factor binding sites in its promoter region ${ }^{19}$, and an Ets-1 blockade reversed the upregulation of $\alpha 5$ and $\beta 1$ integrins in ${ }^{\mathrm{mob}} \mathrm{PBSCs}$ by COMP-Ang1. Considering these results, we postulated that elevated Ets-1 might be responsible for the upregulation of $\alpha 5$ integrin induced by Ang1 after OGD. As expected, the silencing experiment confirmed that knockdown of Ets-1 expression in BECs markedly decreased integrin $\alpha 5$ expression in the presence or absence of Ang1 after OGD. To directly investigate the effect of knockdown of Ets-1 on the upregulation of $\alpha 5$ integrin induced by Ang1 after OGD/ $\mathrm{R}$, we compared the difference between the fold increases of integrin $\alpha 5$ induced by Ang1 in siRNA-Ctl-treated controls and siRNA-Ets-1-treated BECs. This comparison revealed that diminishment of the levels of Ets-1 in BECs significantly reduced the increase rate of integrin $\alpha 5$ induced by Ang1 after OGD compared to the siRNA-Ctltreated controls. These results suggest that the enhanced expression of $\alpha 5$ integrin induced by Ang 1 after OGD/R is dependent on the upregulation of Ets-1.

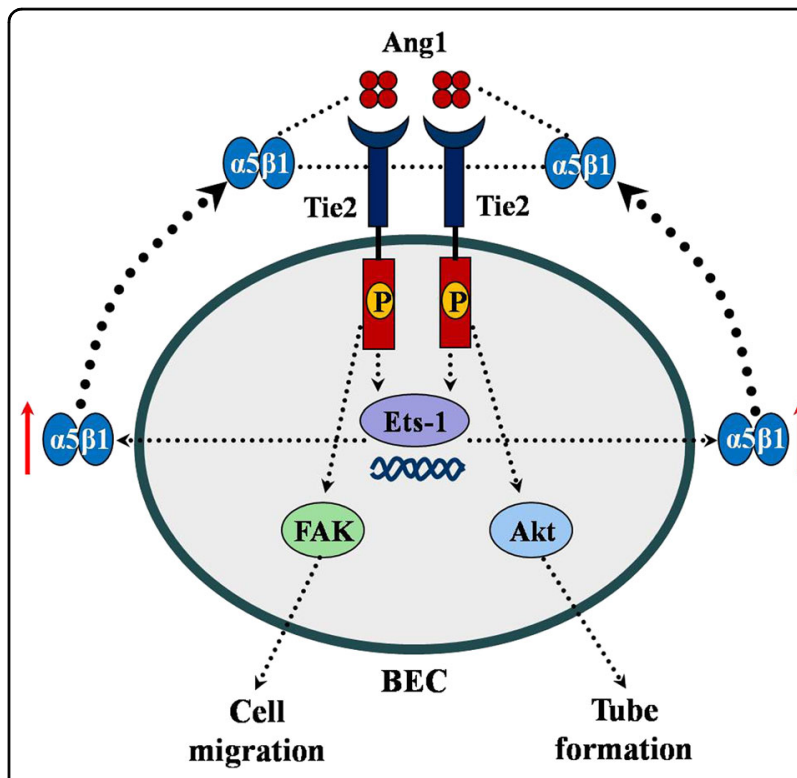

Fig. 8 Schematic representation of Ang1/Tie2 and integrin a5 cooperative signaling in brain endothelial cell regulation.

Following OGD, Ang1/Tie2 is upregulated, which cooperates with integrin a5 to induce Tie2 activation and the phosphorylation of its downstream signaling molecules FAK and Akt in BECs, resulting in the increase of migration and capillary-like tube formation of BECs. Additionally, priming of BECs with Ang1 promotes the expression of a5 integrin through Ets-1 signaling after OGD. Abbreviations: angiopoietin-1, Ang1; brain endothelial cells, BECs; v-ets

erythroblastosis virus E26 oncogene homolog-1, Ets-1; focal adhesion kinase, FAK; phosphorylation, P

\section{Integrin a5 regulation of Ang1-induced migration and tube formation of BECs after OGD}

The Ang1-Tie2-Akt system is believed to be a key regulator of several angiogenic processes, including endothelial cell survival, migration, sprouting, and tube formation ${ }^{15}$. The expression of $\alpha 5 \beta 1$ integrin is upregulated in response to angiogenic factors ${ }^{21}$, and $\alpha 5 \beta 1$ integrin has previously been shown to be involved in mediating cell migration and tube formation in human choroidal endothelial cells ${ }^{16}$. In this study, we further found that Ang1 induces cell migration and tube formation of BECs after OGD, but this effect was attenuated by diminishing the levels of $\alpha 5$ integrin in BECs. Similarly, our previous study and other reports showed that antagonists of $\alpha 5 \beta 1$ integrin blocked tumor necrosis factor- $\alpha, b F G F$, and interleukin-8-stimulated angiogenesis, but had a minimal effect on the vascular endothelial growth factor (VEGF)-A response ${ }^{22,23}$. This evidence indicates that Ang1/Tie2 elicits angiogenesis after cerebral ischemia through strict cooperation with $\alpha 5 \beta 1$ integrin. VEGF has also previously been shown to activate Tie receptors $^{24}$, thus VEGF might be an alternative target to influence Tie 2 activation independently of $\alpha 5 \beta 1$ integrin. Therefore, further studies specifically designed to 
investigate the effects of VEGF and its interrelationship with $\alpha 5 \beta 1$ integrin on Tie2 activation after cerebral ischemia should be done before a conclusion can be reached.

In summary, our results demonstrate that the Ang1/ Tie2 system interacts with integrin $\alpha 5 \beta 1$ on BECs after cerebral ischemia. Specifically, integrin $\alpha 5 \beta 1$ regulates cerebral ischemia-induced BEC activation of Tie2 and priming of BECs with Ang1 via the Tie2/Ets-1 pathway, significantly upregulating the expression of integrin $\alpha 5$. Functionally, integrin $\alpha 5$ regulates Ang1-induced migration and tube formation of BECs after OGD (Fig. 8). Knowledge of these molecular and signaling pathways within BECs may facilitate the design of drugs aimed at promoting angiogenic remodeling immediately after cerebral ischemia and thus contribute to novel vascular protective strategies in the treatment of ischemic stroke.

\section{Acknowledgements}

This study was supported by the National Natural Science Foundation of China (No. 81571203, 81771328), the Shanghai Natural Science Foundation of China (No. 15ZR1437200), the Outstanding Leaders Training Program of Pudong Health Bureau of Shanghai (No. PWR12014-05), the Key Specialty Construction Project of the Shanghai Municipal Commission of Health and Family Planning (No. ZK2015B16), and the Municipal Human Resources Development Program for Outstanding Leaders in Medical Disciplines in Shanghai (No. 2017BR051). All sources of funding for the research declare that they have no competing financial or personal interests and that none of the author's institutions have contracts relating to this research through which they may stand to gain financially now or in the future.

\section{Author details}

'Department of Special Outpatient Service, Gongli Hospital, The Second Military Medical University, Shanghai 200135, P. R. China. ²Department of Neurology, Gongli Hospital, The Second Military Medical University, Shanghai 200135, P. R. China. ${ }^{3}$ The Graduate School, Ningxia Medical University, Yinchuan, Ningxia 750004, P. R. China. ${ }^{4}$ Department of Pharmacy, Gongli Hospital, The Second Military Medical University, Shanghai 200135, P. R. China. ${ }^{5}$ Department of Neurology, Taishan People's Hospital, Taishan 529200, P. R. China. ${ }^{6}$ Department of Molecular Medicine, The Scripps Research Institute, 10550 North Torrey Pines Road, La Jolla, CA 92037, USA

\section{Conflict of interest}

The authors declare that they have no conflict of interest.

\section{Publisher's note}

Springer Nature remains neutral with regard to jurisdictional claims in published maps and institutional affiliations.

Supplementary information accompanies this paper at https://doi.org/ 10.1038/s12276-018-0145-7.

Received: 26 January 2018 Revised: 6 April 2018 Accepted: 14 May 2018. Published online: 5 September 2018

\section{References}

1. Huang, Q. et al. The temporal expression patterns of fibronectin and its receptors-a5 $\beta 1$ and av $\beta 3$ integrins on blood vessels after cerebral ischemia. Restor. Neurol. Neurosci. 33, 493-507 (2015).
2. Li, L., Liu, F., Welser-Alves, J. V., McCullough, L. D. \& Milner, R. Upregulation of fibronectin and the a5 $\beta 1$ and av $\beta 3$ integrins on blood vessels within the cerebral ischemic penumbra. Exp. Neurol. 233, 283-291 (2012).

3. Ruan, L., Wang, B., ZhuGe, Q. \& Jin, K. Coupling of neurogenesis and angiogenesis after ischemic stroke. Brain Res. 1623, 166-173 (2015).

4. Krupinski, J., Kaluza, J., Kumar, P., Kumar, S. \& Wang, J. M. Role of angiogenesis in patients with cerebral ischemic stroke. Stroke 25, 1794-1798 (1994).

5. Chen, J., Venkat, P., Zacharek, A. \& Chopp, M. Neurorestorative therapy for stroke. Front. Hum. Neurosci. 8, 382 (2014).

6. Kim, I. et al. Angiopoietin-1 induces endothelial cell sprouting through the activation of focal adhesion kinase and plasmin secretion. Circ. Res. 86 952-959 (2000).

7. DeBusk, L. M., Hallahan, D. E. \& Lin, P. C. Akt is a major angiogenic mediator downstream of the Ang1/Tie2 signaling pathway. Exp. Cell Res. 298, 167-177 (2004).

8. Moon, H. E. et al. COMP-Ang1 potentiates EPC treatment of ischemic brain injury by enhancing angiogenesis through activating AKT-mTOR pathway and promoting vascular migration through activating Tie2-FAK pathway. Exp. Neurobiol. 24, 55-70 (2015).

9. Sun, J. et al. Vascular expression ofangiopoietin1, a5 $\beta 1$ integrin and tight junction proteins is tightly regulated during vascular remodeling in the postischemic brain. Neuroscience 362, 248-256 (2017).

10. Cascone, I., Napione, L., Maniero, F., Serini, G. \& Bussolino, F. Stable interaction between alpha5beta1 integrin and Tie2 tyrosine kinase receptor regulates endothelial cell response to Ang-1. J. Cell Biol. 170, 993-1004 (2005).

11. Kim, M. S. et al. Priming with angiopoietin-1 augments the vasculogenic potential of the peripheral blood stem cells mobilized with granulocyte colony-stimulating factor through a novel Tie2/Ets-1 pathway. Circulation 120, 2240-2250 (2009).

12. Hwang, B. et al. Stimulation of angiogenesis and survival of endothelial cells by human monoclonal Tie2 receptor antibody. Biomaterials 51, 119-128 (2015).

13. Sheibani, N. \& Frazier, W. A. Thrombospondin 1 expression in transformed endothelial cells restores a normal phenotype and suppresses their tumorigenesis. Proc. Natl Acad. Sci. USA 92, 6788-6792 (1995).

14. Dalton, A. C., Shlamkovitch, T., Papo, N. \& Barton, W. A. Constitutive association of Tie1 and Tie2 with endothelial integrins is functionally modulated by Angiopoietin-1 and fibronectin. PLOS. ONE. 11, e0163732 (2016).

15. Augustin, H. G., Koh, G. Y., Thurston, G. \& Alitalo, K. Control of vascular morphogenesis and homeostasis through the angiopoietin-Tie system. Nat. Rev. Mol. Cell Biol. 10, 165-177 (2009).

16. Wang, W. et al. The antiangiogenic effects of integrin alpha5beta1 inhibitor (ATN-161) in vitro and in vivo. Invest. Ophthalmol. Vis. Sci. 52, 7213-7220 (2011)

17. Zacharek, A. et al. Angiopoietin1/Tie2 and VEGF/Flk1 induced by MSC treatment amplifies angiogenesis and vascular stabilization after stroke. J. Cereb. Blood. Flow. Metab. 27, 1684-1691 (2007).

18. Xia, H., Nho, R. S., Kahm, J., Kleidon, J. \& Henke, C. A. Focal adhesion kinase is upstream of phosphatidylinositol 3-kinase/Akt in regulating fibroblast survival in response to contraction of type I collagen matrices via a beta 1 integrin viability signaling pathway. J. Biol. Chem. 279, 33024-33034 (2004).

19. Sato, Y. Role of ETS family transcription factors in vascular development and angiogenesis. Cell Struct. Funct. 26, 19-24 (2001).

20. Salnikow, K. et al. Regulation of hypoxia-inducible genes by ETS1 transcription factor. Carcinogenesis 29, 1493-1499 (2008).

21. Enaida, H. et al. Effect of growth factors on expression of integrin subtypes in microvascular endothelial cells isolated from bovine retinas. Fukushima. J. Med. Sci. 44, 43-52 (1998).

22. Huang, H., Huang, Q., Wang, F., Milner, R. \& Li, L. Cerebral ischemiainduced angiogenesis is dependent on tumor necrosis factor receptor 1mediated upregulation of a5 $\beta 1$ and aV $\beta 3$ integrins. J. Neuroinflamm. 13, 227 (2016).

23. Kim, S., Bell, K., Mousa, S. A. \& Varner, J. A. Regulation of angiogenesis in vivo by ligation of integrin alpha5beta1 with the central cell-binding domain of fibronectin. Am. J. Pathol. 156, 1345-1362 (2000).

24. Singh, H., Milner, C. S., Aguilar Hernandez, M. M., Patel, N. \& Brindle, N. P. Vascular endothelial growth factor activates the Tie family of receptor tyrosine kinases. Cell Signal. 21, 1346-1350 (2009). 